\title{
International Coordination of Planetary Coordinate System Recommendations and the IAU Working Group on Cartographic Coordinates and Rotational Elements
}

\author{
Brent Archinal $^{\mathrm{a},}$ *, IAU Working Group on Cartographic Coordinates and Rotational Elements \\ ${ }^{a}$ U. S. Geological Survey, 2255 N. Gemini Drive, Flagstaff, AZ 86001, USA, barchinal@usgs.gov \\ * Corresponding author
}

Keywords: Standards, Cartographic coordinates, Coordinate systems, Coordinate frames, Solar System bodies

\begin{abstract}
:
Overview: Approximately every 3 years since 1979, the Working Group on Cartographic Coordinates and Rotational Elements (hereafter the "WGCCRE", https://astrogeology.usgs.gov/groups/IAU-WGCCRE), a functional working group of the International Astronomical Union (IAU), has issued a report following most IAU General Assembly meetings. The report includes recommendations on coordinate systems and related parameters (body orientation and shape) that can be used for making cartographic products (maps) of Solar System bodies. These recommendations, which are open to further modification when indicated by community consensus, facilitate the use and comparison of multiple datasets by promoting the use of an internationally standardized set of mapping parameters.
\end{abstract}

Over the past year and a half, the WGCCRE has been making presentations seeking input about its future. We are looking for input on: 1) how the WGCCRE makes recommendations and on specific new recommendations we are considering based on user input; and 2) the future of the WGCCRE itself, in terms of how it should interact with users and other related groups and its future structure.

Our presentation at the $30^{\text {th }}$ International Cartographic Conference will include a status report on the WGCCRE, summarize our efforts and activities in 2019-2021, and cover our report and correction publication from 2018 and 2019, respectively (Archinal et al., 2018, 2019a)].

In the remainder of this abstract and in much of our planned presentation we look to the future and highlight possible upcoming recommendations from the WGCCRE, consider possible future directions for cooperation with other organizations, and what future form the WGGCRE might take. We end by asking for input on these issues from the International Cartographic Association community.

For further details on the WGCCRE and these issues, we direct the reader to the references just cited, our 2020 paper to the ISPRS Congress (Archinal et al., 2020), and a white paper submitted to the NASA Planetary Science and Astrobiology Decadal Survey 2023-2032 (Paganelli, et al., 2020).

Addressing the future: The WGCCRE began in 1976 and, as part of the IAU, established fundamental principles regarding planetary coordinates and planetary mapping. After 45 years, some obvious questions naturally arise. Are these principles still adequate? Are changes needed? When is any transition or refinement needed in a coordinate system and how should it be done? As one example, for bodies where a longitude definition has been defined from terrestrial observations, when and how should any transition be done based on spacecraft observations? What are the best methods for recording the fundamental parameters defining cartographic coordinates and rotational elements for planetary bodies? What procedures need to be further recommended for establishing and updating these coordinate systems and frames? Does it make sense to switch from the existing widespread use of planetographic systems for planets and satellites to planetocentric coordinate systems? How could conflicting published information on planetary coordinate systems be addressed in a timely way and who would address such conflicts, particularly when such issues often require substantial research?

Operational questions are also key to future WGCCRE activities and should be considered. Does the lunar and planetary community understand the need and value of such recommendations and standards? How, for example, could such activities be better publicized to further such understanding? What methods could improve input from the community? Is a service, perhaps analogous to the International Earth Rotation and Reference Systems Service (IERS; https://www.iers.org/), needed to provide real time support, e.g., addressing issues and questions as they arise, and perhaps more quickly updating coordinate system recommendations? Should it be recommended that journals require the proper use of coordinate system definitions (just as some journals require proper identifications of meteorites)? How should this work be supported and funded? Presumably, the various space agencies involved would have to agree to support such infrastructure, based on the value of improvements possible for science and exploration and increased 
efficiency from the use of improved standards and mapping methods. How important were groups such as the now inactive NASA Mars Geodesy and Cartography Working Group (MGCWG) (Duxbury et al., 2002) and Lunar Geodesy and Cartography Working Group (LGCWG) (Archinal and the LGCWG, 2009)? Do those groups need to be reestablished? Are separate groups needed to address coordinate systems, data formats, and products needed for other bodies (e.g., outer planets, Mercury, small bodies)? How does all this fit into the recently recognized need to develop planetary spatial data infrastructure (Laura et al., 2017), whether overall or for individual bodies?

What types of coordination would be beneficial (and possible) between the existing groups that, to some degree, address the listed issues? These include the IAU itself, other international groups, and the various international space agencies. Because of its long history and number of missions, several NASA-centric groups have addressed these issues in the past and present, although most have included a significant international component. Examples of these many organizations include the WGCCRE; IAU Commission A3 on Fundamental Standards; the IAU Working Group for Planetary System Nomenclature; the International Association of Geodesy (IAG); the ISPRS Planetary Remote Sensing and Mapping working group (https://www2.isprs.org/commissions/comm3/icwg-3-2.html); the International Cartographic Association Commission on Planetary Cartography (https://planetcarto.wordpress.com/); the International Planetary Data Alliance (IPDA, https://planetarydata.org/); the NASA Mapping And Planetary Spatial Infrastructure Team (MAPSIT (Radebaugh et al., 2019)); the other NASA analysis and assessment groups (https://www.lpi.usra.edu/analysis/); and the NASA Planetary Data System (PDS, https://pds.nasa.gov/). These groups and space agencies are already in communication to varying extents. Are there cases where strengthening of a formalized relationship would be useful or where new connections need to be established? How would any increased activities be staffed and funded? The benefits of such activities are well known among many of those participating in these organizations, but likely would need to be better publicized in terms of the critical support provided for planetary science and exploration, e.g., for allowing for safe navigation, registration and comparison of datasets, and support of landing and surface exploration, operations, and science.

Request for Input: The WGCCRE desires continued input from the planetary community, and at this point input from International Cartographic Association community and in particular the ICA Commission on Planetary Cartography. This includes input regarding both specifics such as the systems for individual bodies and more general questions such as the operation of the WGCCRE. We would particularly like input on the topics and questions presented above. We encourage members of the planetary science and particularly the planetary mapping community to please use the contact information above. We will consider how to go forward both in addressing some of these questions and cited issues, perhaps with appropriate surveys, and discussions at least with some of the key groups mentioned, and key space agency personnel.

We would like our community colleagues to be aware that we regularly provide summaries (such as this one) and make meeting presentations to increase awareness of our work (Archinal et al., 2019b, 2020a, 2020b, 2021a, 2021b). We encourage volunteers to become WGCCRE members and help with our efforts. Our membership is open to IAU members who are willing to indicate their area of expertise and how they plan to help with our reports. Again, please use the contact information above for additional information.

Acknowledgments: Funding for B. Archinal has been provided via a NASA-USGS Interagency Agreement on Planetary Spatial Data Infrastructure.

\section{References}

Archinal et al. (2018) CMDA, 130:22, doi: 10.1007/s10569-017-9805-5.

Archinal et al. (2019a) CMDA, 121:12, doi: 10.1007/s10569-019-9925-1.

Archinal et al. (2019b) IAG Symposium G01, \#IUGG19-1604.

Archinal et al. (2020a) ISPRS Arch. Photogram., XLIII-B3-2020, 1091-1097, https://www.int-arch-photogrammremote-sens-spatial-inf-sci.net/XLIII-B3-2020/1091/2020/.

Archinal et al. (2020b) LPS LI, \#2385.

Archinal et al. (2021a) 43rd COSPAR, \#PSD.1-0003-21.

Archinal et al. (2021b) $5^{\text {th }} P D W$ and $2^{\text {nd }} P S I D A, \# 7051$.

Archinal and the LGCWG (2009) LPS XL, \#2095.

Duxbury et al. (2002) ISPRS XXXIV, Part 4, https://www.isprs.org/proceedings/XXXIV/part4/pdfpapers/521.pdf.

Laura et al. (2017) ISPRS Journal, 6, 181, doi: 10.3390/ijgi6060181.

Paganelli et al. (2020) White paper submission to Planetary Science and Astrobiology Decadal Survey 2023-2032, https://is.gd/WGCCRE2020wp.

Radebaugh et al. (2019) EPSC-DPS Meeting, \#EPSC-DPS2019-951. 